\section{Cobertura e fatores associados à realização do exame de detecção do câncer de colo de útero em área urbana no Sul do Brasil: estudo de base populacional}

\author{
Cervical cancer screening coverage and associated \\ factors in a city in southern Brazil: a population- \\ based study
}

\author{
1 Universidade do Vale do \\ Itajaí, Itajaí, Brasil. \\ 2 Programa de Pós- \\ graduação em Saúde \\ Coletiva, Universidade \\ Federal de Santa Catarina, \\ Florianópolis, Brasil. \\ Correspondência \\ S. I. Gasperin \\ Universidade do Vale do Itajaí. \\ Rua Uruguai 458, Itajaí, \\ SC 88302-202, Brasil. \\ simoneg@univali.br
}

\begin{abstract}
This study focused on coverage of screening for cervical cancer and associated factors in women 20 to 59 years of age in Florianópolis, Santa Catarina State, Brazil, in 2009. This was a crosssectional study using cluster random sampling. Two outcomes were examined: a history of at least one Papanicolaou test and delaying the test (never performed or performed more than three years previously). Among 952 women, 93\% (95\%CI: 91.5-94.7) had ever had a Pap smear, whereas 14\% (95\%CI: 11.8-16.2) had delayed the test. According to Poisson regression, both outcomes were associated with marital status, schooling, presence of chronic disease, and consulting a physician for other reasons. Age, income, and hospitalization in the previous year were only associated with ever having a Pap smear. Although test coverage was high, much of the screening was opportunistic. In addition, various social, economic, and demographic disparities influenced the odds of being screened.
\end{abstract}

Vaginal Smears; Uterine Cervical Neoplasms; Health Services Coverage; Mass Screening
Simone Iara Gasperin 1,2

Antonio Fernando Boing 2

Emil Kupek ${ }^{2}$

\section{Introdução}

As neoplasias representaram, no início do século XXI, um problema de saúde pública em todo o mundo. A Organização Mundial da Saúde (OMS) estima que, em 2007, ocorreram 12 milhões de casos novos e, aproximadamente, 7,9 milhões de mortes por câncer, representando $13 \%$ de todos os óbitos no mundo. Para o ano de 2030, são esperados cerca de 26 milhões de casos novos e 17 milhões de mortes pela doença 1,2 .

Entre as mulheres, as neoplasias de mama e colo do útero são os tipos mais frequentes. Calcula-se que, em 2008, surgiram 529 mil casos novos de câncer de colo do útero em todo o mundo, com taxa de mortalidade de 7,8 a cada 100 mil mulheres ${ }^{2}$. No Brasil, em 2010, estima-se a ocorrência de 18 casos novos de câncer de colo de útero a cada 100 mil mulheres 3 .

Essa neoplasia tem um prognóstico bom quando diagnosticada e tratada precocemente ${ }^{4}$. A detecção precoce do câncer de colo do útero faz parte das ações de prevenção secundária, cuja principal estratégia é o rastreamento de mulheres sexualmente ativas por meio do exame citopatológico do colo uterino ou teste de Papanicolaou 5 . A efetividade desse programa está relacionada com taxas de cobertura maiores que $80 \%$. Avalia-se que essa cobertura possa diminuir pela metade a mortalidade por câncer cervical 6,7 . 
O Ministério da Saúde do Brasil, desde 1988, segue as recomendações da OMS, a qual preconiza, para a detecção precoce dessa neoplasia, a realização do teste de Papanicolaou em mulheres de 25 a 59 anos de idade com uma periodicidade trienal após dois exames anuais consecutivos negativos 6 . No entanto, no Brasil, ainda predominam os exames realizados de forma oportunística, com a procura espontânea dos serviços de saúde por razões diversas que não a prevenção. Em consequência, a metade dos casos é diagnosticada em estádios avançados da doença, mantendo elevada a taxa de mortalidade há duas décadas, sem evidências de reduções significativas 4,8 .

Poucas publicações científicas evidenciam a cobertura do teste de Papanicolaou e os fatores associados à sua realização no Brasil em nível populacional. Um inquérito domiciliar realizado em 15 capitais brasileiras e no Distrito Federal identificou que as prevalências de realização do teste nos últimos três anos variaram de 73,4\% a $92,9 \%$. Dentre os fatores associados à não realização desta prática preventiva pelas mulheres, destacaram-se a idade mais avançada, cor preta ou parda, baixa escolaridade e menor renda familiar per capita, revelando desigualdades na cobertura 9,10,11.

Os estudos populacionais permitem identificar as desigualdades na cobertura do exame de prevenção do câncer de colo do útero, pois possibilitam distinguir as mulheres que fizeram apenas um teste daquelas que fizeram vários; além disso, investigam aquelas que realizam os exames fora do sistema público de saúde. Esse tipo de pesquisa é fundamental para estimar o impacto das ações desenvolvidas e permite o monitoramento e a avaliação do programa, contribuindo para o planejamento e organização dos serviços de saúde.

O objetivo deste estudo foi conhecer a cobertura do teste de Papanicolaou na população feminina residente na área urbana do Município de Florianópolis, Santa Catarina, Brasil, e também identificar a proporção de exames não realizados ou em atraso. O estudo analisou, ainda, fatores demográficos e socioeconômicos, além de condições e comportamentos de saúde associados à realização deste exame.

\section{Metodologia}

Trata-se de um estudo transversal de base populacional, realizado com mulheres adultas (20 a 59 anos de idade), residentes, em 2009, na zona urbana de Florianópolis, capital do Estado de Santa Catarina. A pesquisa é integrante do Projeto
EpiFloripa Adulto, que investigou as condições de vida e saúde da população dessa faixa etária entre setembro de 2009 e janeiro de 2010.

Florianópolis é uma cidade de médio porte, com população estimada, para o ano de 2009, de 408.163 habitantes. Deste total, 129.035 eram mulheres adultas, representando $31,6 \%$ de toda a população do município (http://tabnet.data sus.gov.br/cgi/tabcgi.exe?ibge/cnv/popsc.def/, acessado em 02/Mai/2010).

A amostra foi calculada por intermédio do programa Epi Info 6.04 (Centers for Disease Control and Prevention, Atlanta, Estados Unidos), considerando-se nível de $95 \%$ de confiança, poder de $80 \%$, erro máximo de $3,5 \%$ e prevalência de realização do exame de detecção precoce do câncer de colo uterino de 85\%. Considerou-se, ainda, efeito de delineamento igual a 2, adicionando-se $10 \%$ por potenciais perdas e recusas e $15 \%$ para controle de fatores de confusão. A amostra final foi de 1.010 mulheres.

O processo de amostragem deu-se por conglomerados, em dois estágios, considerando-se como unidades de primeiro estágio os setores censitários. Estes foram estratificados em decis, de acordo com a renda do chefe da família, e sorteados de forma sistemática, selecionando-se seis setores em cada decil de renda, num total de 60 setores censitários dentre os 420 existentes na zona urbana do município. Todos os setores sorteados foram visitados pela equipe de campo, realizando-se a contagem dos domicílios residenciais ocupados, que foram considerados como unidades de segundo estágio. Uma vez que o número de domicílios entre os setores variou de 61 a 810, realizaram-se fusões e divisões de setores, respeitando-se a localização geográfica e o decil de renda de cada um. Procurou-se, assim, reduzir a variação no número de domicílios entre os setores censitários. Dos 17.755 domicílios elegíveis nos 60 setores censitários, foram sorteados 1.134 para a pesquisa.

Todas as mulheres adultas moradoras dos domicílios sorteados eram convidadas a participar do estudo, não sendo aceitos terceiros como respondentes. As perdas foram consideradas somente após quatro visitas sem que o entrevistador conseguisse localizar o morador (sendo uma no final de semana e outra no horário noturno). As recusas ocorreram quando a pessoa optou por não participar do estudo.

Os dados foram obtidos por meio de um questionário estruturado em blocos temáticos, pré-testado com trinta adultos, antes da coleta dos dados. Também foi realizado estudo-piloto em setores censitários não incluídos no estudo. As entrevistadoras, todas do sexo feminino, apresentavam como formação mínima Ensino Médio 
completo e disponibilidade de tempo integral para o trabalho de campo. Todas foram treinadas pelos coordenadores e supervisores do estudo para a aplicação do questionário e para a coleta de medidas antropométricas.

No processo de coleta dos dados, utilizou-se um computador de dimensões reduzidas, o Personal Digital Assistant (PDA), eliminando a etapa de digitação e possíveis erros decorrentes desta. Os questionários eletrônicos foram coletados semanalmente e organizados em um banco de dados. As informações incompletas ou inconsistentes foram corrigidas em segunda entrevista, quando necessário.

Dois aspectos da cobertura do teste de $\mathrm{Pa}$ panicolaou foram investigados: (1) prevalência e fatores associados à realização do exame pelo menos uma vez na vida e (2) prevalência e fatores associados ao exame em atraso. Esta situação foi assim caracterizada: exame nunca realizado na vida ou quando realizado há mais de três anos, de acordo com o foco do programa de prevenção precoce do câncer de colo de útero estabelecido pelo Ministério da Saúde 6 .

A fim de evitar viés de informação, investigouse o conhecimento das mulheres sobre o teste de Papanicolaou. Aquelas que informaram nunca ter ouvido falar do exame ( $2 \%$ das mulheres investigadas) não responderam às demais perguntas do questionário e considerou-se que nunca realizaram o exame.

As variáveis independentes analisadas foram:

- Demográficas e socioeconômicas: idade (em categorias de 10 anos), cor/raça autorreferida (branca, parda ou preta), situação conjugal (casada/com companheiro, solteira, divorciada/viúva), escolaridade (anos completos de estudo, categorizados em até 4, 5-8, 9-11 e 12 ou mais), renda familiar mensal per capita (em tercis) e situação ocupacional (exercer trabalho remunerado no momento da entrevista);

- Condições de saúde: presença de pelo menos uma doença crônica autorreferida; autoavaliação de saúde, caracterizada como positiva (boa ou muito boa) ou negativa (regular, ruim ou muito ruim); presença de sobrepeso ou obesidade, calculados com base nas medidas do índice de massa corporal, sendo valores iguais ou superiores a $25 \mathrm{~kg} / \mathrm{m}^{2}$ classificados como sobrepeso ou obesidade;

- Comportamentos de saúde: tabagismo (fumante, ex-fumante e nunca fumou); uso abusivo de álcool, mensurado por meio do Alcohol Use Disorders Identification Test (AUDIT) 12, com ponto de corte superior a 7 pontos; prática de atividade física, caracterizada pela realização de exercício físico ou esporte nos últimos três meses;
- Uso dos serviços de saúde: possuir ou não plano privado de saúde; realização de consulta médica nos últimos 15 dias; internação hospitalar; visita do agente comunitário de saúde nos 12 meses que antecederam a entrevista; tipo de serviço utilizado para realização dos exames preventivos de câncer (público ou privado).

Os dados foram analisados no programa Stata 9 (Stata Corp., College Station, Estados Unidos). As análises bivariadas incluíram estimativas de prevalência dos dois desfechos, seus respectivos intervalos de 95\% de confiança (IC95\%), valores de $\mathrm{p}$ do teste de qui-quadrado de Pearson e teste de tendência linear quando oportuno. Na análise das associações, apesar de os desfechos estudados serem binários, optou-se pela regressão de Poisson, estimando-se razões de prevalência (RP). Também consideraram-se na análise, a estratégia amostral, o efeito de delineamento e os pesos individuais, utilizando-se o comando "svy" no Stata. O cálculo dos respectivos intervalos de confiança e a análise de significância estatística (valor de p) foram obtidos por meio do teste de Wald. O critério para inclusão de variáveis no modelo múltiplo foi o valor $\mathrm{p} \leq 0,20$ na análise bivariada. Para sua permanência no modelo final, considerou-se o valor de $\mathrm{p} \leq 0,05$. O modelo de regressão de Poisson foi construído com a entrada das variáveis passo a passo, ordenadas do menor para o maior valor de $\mathrm{p}$.

O projeto de pesquisa foi aprovado pelo Comitê de Ética em Pesquisa com Seres Humanos da Universidade Federal de Santa Catarina (UFSC), sob parecer 351/08. Aos indivíduos que concordaram em participar do estudo foi garantido sigilo das informações após a assinatura do termo de consentimento livre e esclarecido.

\section{Resultados}

Foram entrevistadas 952 mulheres com idade entre 20 e 59 anos das 1.010 definidas no cálculo de amostra (equivalente a $94,2 \%$ ). A maior parte delas (52\%) tinha idade entre 20 e 39 anos, sendo a média de idade igual a 38,6 anos. A maioria das entrevistadas relatou ser da cor branca $(86,4 \%)$, estar casada ou viver em união estável (61\%), ter nove ou mais anos de estudo (75,5\%) e exercer trabalho remunerado $(70,9 \%)$ (Tabela 1$)$.

Quanto à realização do teste de Papanicolaou, 93\% (IC95\%: 91,5-94,7) das mulheres investigadas informaram ter realizado o exame pelo menos uma vez na vida, e 14\% (IC95\%: 11,8-16,2) estavam com o exame em atraso, com igual proporção (7\%; IC95\%: 5,3-8,6) para exame nunca realizado ou realizado há mais de três anos. 
Prevalência de realização do exame de Papanicolau na vida e exames em atraso segundo variáveis demográficas e socioeconômicas, em 952 mulheres de 20 a 59 anos. Florianópolis, Santa Catarina, Brasil, 2009.

\begin{tabular}{|c|c|c|c|c|c|}
\hline \multirow[t]{2}{*}{ Variáveis } & \multirow[t]{2}{*}{ n (\%) } & \multicolumn{2}{|c|}{ Cobertura na vida } & \multicolumn{2}{|c|}{ Exames em atraso } \\
\hline & & $\begin{array}{l}\text { Prevalência\% } \\
\text { (IC95\%) }\end{array}$ & Valor de $p$ * & $\begin{array}{l}\text { Prevalência \% } \\
\text { (IC95\%) }\end{array}$ & Valor de $p^{*}$ \\
\hline Idade (anos) & & & $<0,001$ ** & & 0,049 \\
\hline $20-29$ & $278(29,2)$ & $83,5(78,0-88,9)$ & & $18,7(13,1-24,3)$ & \\
\hline $30-39$ & $217(22,8)$ & $96,8(94,1-99,4)$ & & $11,1(6,1-16,0)$ & \\
\hline $40-49$ & $256(26,9)$ & $96,9(94,6-99,2)$ & & $11,7(7,4-16,1)$ & \\
\hline $50-59$ & $201(21,1)$ & $97,5(95,4-99,6)$ & & $13,4(8,3-18,6)$ & \\
\hline Cor da pele autorreferida $\star \star \star \star$ & & & 0,001 & & 0,004 \\
\hline Branca & $796(86,4)$ & $94,1(91,6-96,6)$ & & $12,6(9,3-15,8)$ & \\
\hline Parda & $72(7,8)$ & $91,7(84,6-98,7)$ & & $16,7(7,0-26,4)$ & \\
\hline Preta & $53(5,8)$ & $81,1(68,0-94,3)$ & & $28,3(13,2-43,4)$ & \\
\hline Estado civil & & & $<0,001$ & & $<0,001$ \\
\hline Casada/União estável & $581(61,0)$ & $95,9(93,6-98,1)$ & & $11,0(7,8-14,3)$ & \\
\hline Solteira & $248(26,0)$ & $84,7(78,9-90,4)$ & & $22,6(16,3-28,9)$ & \\
\hline Divorciada/Separada/Viúva & $123(13,0)$ & $96,7(93,6-99,8)$ & & $10,6(5,2-15,9)$ & \\
\hline Escolaridade (anos de estudo) & & & $<0,001$ & & $<0,001 * *$ \\
\hline 12 ou mais & $416(43,7)$ & $97,1(95,5-98,8)$ & & $7,4(5,3-9,5)$ & \\
\hline $9-11$ & $302(31,8)$ & $89,1(84,9-93,2)$ & & $15,9(11,0-20,8)$ & \\
\hline $5-8$ & $144(15,1)$ & $91,0(85,8-96,1)$ & & $18,7(12,7-24,8)$ & \\
\hline 4 ou menos & $89(9,4)$ & $91,0(86,1-95,9)$ & & $30,3(20,9-39,8)$ & \\
\hline Renda per capita (em tercis) & & & $<0,001 \star \star *$ & & $<0,001 * *$ \\
\hline 1ㅇ (menor renda) & $312(33,3)$ & $86,5(81,6-91,4)$ & & $22,7(16,8-28,7)$ & \\
\hline 2 & $315(33,7)$ & $95,6(93,1-98,0)$ & & $9,8(6,3-13,4)$ & \\
\hline 3o (maior renda) & $308(33,0)$ & $97,4(95,6-99,2)$ & & $9,4(5,5-13,3)$ & \\
\hline Trabalho remunerado (últimos 30 dias) & & & 0,823 & & 0,376 \\
\hline Não & $277(29,1)$ & $92,8(89,8-95,7)$ & & $15,5(11,1-19,9)$ & \\
\hline Sim & $675(70,9)$ & $93,2(90,3-96,1)$ & & $13,3(9,5-17,2)$ & \\
\hline
\end{tabular}

* Valor de $p$ do teste $\chi^{2}$;

** Valor de $p$ do teste $\chi^{2}$ de tendência linear;

*** Excluídas as categorias amarela e indígena, além dos casos ignorados $(n=921)$.

Observou-se maior cobertura do exame na vida à medida que aumentou a renda, a idade $\mathrm{e}$ a escolaridade, com diferenças estatisticamente significativas $(\mathrm{p}<0,001)$. Já a prevalência de exames em atraso foi significativamente maior para as mulheres com até quatro anos de escolaridade $(30,3 \%)$, que se autorreferiram da cor preta $(28,3 \%)$, com menor renda familiar per capita $(22,7 \%)$ e solteiras $(22,6 \%)$. Em relação à faixa etária, a maior proporção de exames em atraso foi encontrada naquelas com idade entre 20 a 29 anos (18,7\%) e entre 50 a 59 anos (13,4\%); para as mais jovens, o atraso foi caracterizado principalmente por nunca ter realizado o exame, já para as mais velhas, o atraso foi, sobretudo, resultante de um intervalo superior a três anos para a repetição do exame (Tabela 1).
Na Tabela 2, verifica-se que a maior prevalência de cobertura do Papanicolaou na vida foi encontrada entre as mulheres que informaram apresentar duas ou mais doenças crônicas (97,5\%). Já para exames em atraso, as maiores proporções ocorreram no grupo de mulheres sem plano privado de assistência à saúde $(21,1 \%)$, com diferença estatisticamente significante em ambas as situações. As mulheres que haviam tido consulta médica nos 15 dias anteriores à entrevista apresentaram maior cobertura na vida $(91,5 \%)$ e menor proporção de exames em atraso $(8,6 \%)$. Todas as que estiveram internadas em hospital no último ano haviam realizado o exame pelo menos uma vez na vida. Ressalta-se que mais da metade $(61,1 \%)$ das mulheres que passaram por internação hospitalar e cerca de $50 \%$ daquelas 
Tabela 2

Prevalência de realização do exame de Papanicolau na vida e exames não realizados nos últimos três anos, de acordo com comportamentos e condições de saúde, uso dos serviços de saúde, em 952 mulheres de 20 a 59 anos. Florianópolis, Santa Catarina, Brasil, 2009.

\begin{tabular}{|c|c|c|c|c|c|}
\hline \multirow[t]{2}{*}{ Variáveis } & \multirow[t]{2}{*}{ n (\%) } & \multicolumn{2}{|c|}{ Cobertura na vida } & \multicolumn{2}{|c|}{ Exames em atraso } \\
\hline & & $\begin{array}{l}\text { Prevalência \% } \\
\text { (IC95\%) }\end{array}$ & Valor de $p$ * & $\begin{array}{l}\text { Prevalência \% } \\
\text { (IC95\%) }\end{array}$ & Valor de $p$ * \\
\hline \multicolumn{6}{|l|}{ Condições/Comportamentos de saúde } \\
\hline Autoavaliação de saúde & & & 0,821 & & 0,243 \\
\hline Positiva & $732(76,89)$ & $93,2(90,6-95,7)$ & & $13,2(10,0-16,5)$ & \\
\hline Negativa & $220(23,1)$ & $92,7(89,5-96,0)$ & & $16,4(11,9-20,8)$ & \\
\hline Doenças crônicas autorreferidas & & & $<0,001 * *$ & & $0,001 * \star$ \\
\hline Nenhuma & $283(29,7)$ & $87,3(82,1-92,4)$ & & $19,1(13,3-24,9)$ & \\
\hline 1 & $276(29,0)$ & $92,7(89,1-96,4)$ & & $13,8(9,2-18,3)$ & \\
\hline 2 ou mais & $393(41,3)$ & $97,5(96,0-98,9)$ & & $10,4(7,2-13,7)$ & \\
\hline 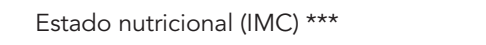 & & & 0,137 ** & & 0,334 ** \\
\hline$<25$ & $516(56,3)$ & $92,0(89,1-95,1)$ & & $13,6(9,7-17,4)$ & \\
\hline$\geq 25<30$ (Sobrepeso) & $246(26,9)$ & $93,5(90,4-96,6)$ & & $14,2(9,3-19,1)$ & \\
\hline$\geq 30$ (Obesidade) & $154(16,8)$ & $95,4(91,8-99,1)$ & & $16,9(10,4-23,4)$ & \\
\hline Tabagismo & & & 0,275 & & 0,192 \\
\hline Nunca fumou & $544(57,2)$ & $92,3(89,3-95,3)$ & & $13,4(9,9-17,0)$ & \\
\hline Ex-fumante & $237(24,9)$ & $95,4(92,4-98,3)$ & & $12,2(7,5-17,0)$ & \\
\hline Fumante & $170(17,9)$ & $92,3(88,5-96,2)$ & & $18,2(10,6-25,9)$ & \\
\hline Uso nocivo de álcool & & & 0,989 & & 0,549 \\
\hline Não & $865(90,9)$ & $93,1(90,4-95,7)$ & & $13,8(10,4-17,1)$ & \\
\hline Sim & $87(9,1)$ & $93,1(86,7-99,5)$ & & $16,1(7,5-24,7)$ & \\
\hline Atividade física (últimos 3 meses) & & & 0,117 & & 0,003 \\
\hline Não & $517(54,4)$ & $91,9(88,7-95,0)$ & & $17,0(13,0-21,0)$ & \\
\hline $\operatorname{Sim}$ & $434(45,6)$ & $94,5(91,9-97,1)$ & & $10,4(7,3-13,4)$ & \\
\hline \multicolumn{6}{|l|}{ Uso dos serviços de saúde } \\
\hline Plano de saúde & & & $<0,001$ & & $<0,001$ \\
\hline Não & $374(39,4)$ & $88,0(83,8-92,2)$ & & $21,1(15,9-26,4)$ & \\
\hline Sim & $576(60,6)$ & $96,5(94,6-98,4)$ & & $9,2(6,2-12,2)$ & \\
\hline Consulta médica (últimos 15 dias) & & & 0,008 & & 0,001 \\
\hline Não & $636(66,9)$ & $91,5(88,6-94,4)$ & & $16,7(12,6-20,7)$ & \\
\hline Sim & $315(33,1)$ & $96,2(94,2-98,2)$ & & $8,6(5,4-11,7)$ & \\
\hline Visita do ACS (últimos 12 meses) \# & & & 0,682 & & 0,420 \\
\hline Não & $642(67,9)$ & $93,5(90,9-96,0)$ & & $13,2(10,0-16,4)$ & \\
\hline Sim & $303(32,1)$ & $92,7(88,9-97,1)$ & & $15,2(9,5-20,8)$ & \\
\hline Internação hospitalar (últimos 12 meses) & & & 0,016 & & 0,278 \\
\hline Não & $879(92,4)$ & $92,5(89,9-95,1)$ & & $14,3(10,9-17,7)$ & \\
\hline Sim & $72(7,6)$ & 100,0 & & $9,7(2,1-17,4)$ & \\
\hline
\end{tabular}

ACS: agente comunitário de saúde; IMC: índice de massa corporal.

* Valor de $p$ do teste $\chi^{2}$;

** Valor de $p$ do teste $\chi^{2}$ de tendência linear;

$\star \star * \star n=916$;

$\# \mathrm{n}=945$. 
que haviam consultado médico relataram apresentar duas ou mais doenças crônicas.

Ainda na Tabela 2, evidencia-se que a prática de atividade física apresentou associação significativa somente para o desfecho de exames em atraso, revelando maior frequência (17\%) entre as mulheres que não realizaram atividade física. Embora a diferença não tenha sido significativa, observou-se maior cobertura do Papanicolaou e menor prevalência de exames em atraso entre as mulheres que informaram não ter recebido a visita do agente comunitário de saúde no último ano.

Na análise das associações entre os desfechos e as variáveis independentes, verificou-se que trabalho remunerado nos últimos trinta dias, tabagismo, uso abusivo de álcool e visita do agente comunitário de saúde no último ano não apresentaram associação estatística para ambos os desfechos na análise bivariada.

A Tabela 3 apresenta os valores brutos e ajustados das razões de prevalência da realização de teste de Papanicolaou na vida e de exames em atraso. Na análise bruta, verificou-se que a distribuição etária, nas faixas de 40 a 49 anos e 50 a 59 anos, foi o fator mais fortemente associado à cobertura do teste de Papanicolaou na vida ( $\mathrm{RP}=$ 1,16; IC95\% 1,09-1,24), com discreta redução na razão de prevalência quando ajustado pelas demais variáveis independentes no modelo final. Analogamente, o fator mais expressivo para o desfecho de exames em atraso foi a baixa escolaridade; as mulheres com quatro anos ou menos de estudo apresentaram prevalência quatro vezes maior em comparação com o grupo de referência, com aumento deste valor após o ajuste final.

$\mathrm{Na}$ análise bruta, as mulheres da cor de pele preta e as solteiras, quando comparadas com as brancas e casadas, apresentaram, para a cobertura do Papanicolaou na vida, razão de prevalência igual a 0,86 (IC95\%: 0,73-1,00) e 0,88 (IC95\%: $0,82-0,94)$, respectivamente. Já para os exames em atraso, ambas as categorias apresentaram prevalência duas vezes maior. Após a análise ajustada, a cor da pele preta deixou de estar significativamente associada aos dois desfechos estudados, permanecendo apenas a associação do estado civil solteira com os exames em atraso.

Quanto às condições e comportamentos de saúde, o relato da ocorrência de duas ou mais doenças crônicas, em comparação com nenhuma doença crônica relatada, foi o único fator associado aos dois desfechos, com discreta redução na força de associação após o ajuste.

No que se refere às características de uso dos serviços de saúde, apesar de a população estudada apresentar elevada cobertura de assistência por plano de saúde privado (60,6\%), a posse de plano esteve associada com os dois desfechos somente na análise bruta, perdendo sua significância estatística no modelo final. Por sua vez, o fato de ter consultado médico na quinzena anterior à coleta dos dados permaneceu positiva e estatisticamente associado com a cobertura na vida e inversamente associado com os exames em atraso, mesmo após ajuste para as demais variáveis independentes. A internação hospitalar no último ano apresentou fraca força de associação com a realização do teste na vida.

Assim, após a análise ajustada, mostraram-se significativamente associados aos dois desfechos os seguintes fatores: estado civil, escolaridade, doenças crônicas autorreferidas e consulta com médico nos últimos 15 dias. As variáveis idade, renda familiar per capita e internação hospitalar no último ano permaneceram associadas somente com a realização do Papanicolaou na vida (Tabela 3).

\section{Discussão}

Os resultados do presente estudo mostraram que 93\% das mulheres entre 20 e 59 anos de idade realizaram pelo menos um teste de Papanicolaou na vida, e a cobertura deste exame nos últimos três anos foi de $86 \%$. Dessa forma, foi alcançada a meta estabelecida pelo Programa Nacional de Prevenção do Câncer Cervical, que propõe cobertura de $80 \%$ da população feminina com um teste a cada três anos 6 .

Por se tratar de um inquérito populacional, este estudo permite a identificação da cobertura real do exame de Papanicolaou e não apenas o número de exames realizados no sistema público de saúde. Outros pontos positivos estão relacionados aos aspectos metodológicos, como a alta taxa de resposta, que foi semelhante em todos os estratos de renda dos setores censitários, e a composição por faixa etária, sendo os valores obtidos no presente estudo bastante semelhantes aos das estimativas do Instituto Brasileiro de Geografia e Estatística (IBGE) para a população adulta de Florianópolis em 2009 (http://tabnet. datasus.gov.br/cgi/tabcgi.exe?ibge/cnv/popsc. def/, acessado em 02/Mai/2010). As perdas apresentaram igualmente distribuição uniforme nos decis de renda familiar, contribuindo para a inferência da amostra.

As limitações dos estudos transversais precisam ser consideradas, principalmente o viés de informação e viés recordatório, condicionados ao autorrelato da realização do Papanicolaou. Algumas mulheres podem não diferenciar o exame ginecológico da coleta de material para 
Tabela 3

Análise bruta e ajustada da realização do teste de Papanicolau na vida e exames em atraso, em 952 mulheres de 20 a 59 anos. Florianópolis, Santa Catarina, Brasil, 2009

\begin{tabular}{|c|c|c|c|c|c|c|}
\hline \multirow[t]{3}{*}{ Variáveis } & \multicolumn{3}{|c|}{ Cobertura na vida } & \multicolumn{3}{|c|}{ Exames em atraso } \\
\hline & Bruta & Ajustada & Valor de $p$ * & Bruta & & Valor de $p$ * \\
\hline & RP (IC95\%) & RP (IC95\%) & & RP (IC95\%) & RP (IC95\%) & \\
\hline \multicolumn{7}{|l|}{ Idade (anos) } \\
\hline $20-29$ & 1,00 & 1,00 & $<0,001$ & 1,00 & 1,00 & $0,159 * \star$ \\
\hline $30-39$ & $1,15(1,08-1,23)$ & $1,09(1,03-1,15)$ & & $0,59(0,35-0,99)$ & $0,88(0,54-1,43)$ & \\
\hline $40-49$ & $1,16(1,09-1,23)$ & $1,09(1,03-1,15)$ & & $0,62(0,41-0,93)$ & $0,87(0,52-1,45)$ & \\
\hline $50-59$ & $1,16(1,09-1,24)$ & $1,10(1,03-1,16)$ & & $0,71(0,47-1,08)$ & $1,02(0,59-1,77)$ & \\
\hline \multicolumn{7}{|c|}{ 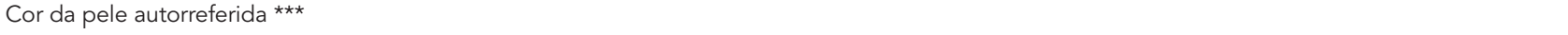 } \\
\hline Branca & 1,00 & 1,00 & 0,228 ** & 1,00 & 1,00 & $0,298 \star \star$ \\
\hline Parda & $0,97(0,89-1,05)$ & $1,00(0,92-1,08)$ & & $1,32(0,72-2,42)$ & $1,10(0,63-1,91)$ & \\
\hline Preta & $0,86(0,73-1,00)$ & $0,92(0,79-1,06)$ & & $2,25(1,27-3,98)$ & $1,19(0,71-2,01)$ & \\
\hline \multicolumn{7}{|l|}{ Estado civil } \\
\hline Casada/União estável & 1,00 & 1,00 & 0,001 & 1,00 & 1,00 & 0,030 \\
\hline Solteira & $0,88(0,82-0,94)$ & $0,92(0,86-0,97)$ & & $2,04(1,46-2,86)$ & $2,36(1,67-3,33)$ & \\
\hline Divorciada/Viúva & $1,00(0,98-1,03)$ & $0,99(0,96-1,02)$ & & $0,95(0,57-1,61)$ & $0,87(0,52-1,47)$ & \\
\hline \multicolumn{7}{|c|}{ Escolaridade (anos de estudo) } \\
\hline 12 ou mais & 1,00 & 1,00 & 0,003 & 1,00 & 1,00 & $<0,001$ \\
\hline $9-11$ & $0,91(0,87-0,96)$ & $0,93(0,90-0,96)$ & & $2,13(1,40-3,24)$ & $1,98(1,28-3,08)$ & \\
\hline $5-8$ & $0,93(0,88-0,98)$ & $0,93(0,88-0,98)$ & & $2,51(1,65-3,81)$ & $2,54(1,53-4,21)$ & \\
\hline 4 ou menos & $0,93(0,88-0,99)$ & $0,93(0,87-0,98)$ & & $4,07(2,82-5,86)$ & $4,74(2,70-8,31)$ & \\
\hline \multicolumn{7}{|l|}{ Renda per capita (em tercis) } \\
\hline 1으 (< renda) & 1,00 & 1,00 & 0,024 & 1,00 & 1,00 & $0,320 * \star$ \\
\hline 2o & $1,10(1,04-1,16)$ & $1,07(1,01-1,13)$ & & $0,43(0,29-0,64)$ & $0,60(0,40-0,89)$ & \\
\hline 3으 (> renda) & $1,12(1,06-1,19)$ & $1,06(1,00-1,11)$ & & $0,41(0,25-0,67)$ & $0,78(0,45-1,33)$ & \\
\hline \multicolumn{7}{|c|}{ Doenças crônicas autorreferidas } \\
\hline Nenhuma & 1,00 & 1,00 & 0,010 & 1,00 & 1,00 & 0,005 \\
\hline 1 & $1,06(1,00-1,12)$ & $1,02(0,97-1,07)$ & & $0,72(0,46-1,11)$ & $0,90(0,59-1,37)$ & \\
\hline 2 ou mais & $1,11(1,05-1,17)$ & $1,06(1,01-1,11)$ & & $0,54(0,39-0,75)$ & $0,62(0,44-0,87)$ & \\
\hline \multicolumn{7}{|l|}{ Estado nutricional (IMC) \# } \\
\hline$<25$ & 1,00 & \#\# & & 1,00 & $\# \#$ & \\
\hline$\geq 25<30$ & $1,01(0,97-1,05)$ & & & $1,04(0,73-1,49)$ & & \\
\hline$\geq 30$ & $1,03(1,00-1,07)$ & & & $1,24(0,83-1,85)$ & & \\
\hline \multicolumn{7}{|c|}{ Atividade física (últimos 3 meses) } \\
\hline Não & 1,00 & \#\# & & 1,00 & $\# \#$ & \\
\hline Sim & $1,02(0,99-1,06)$ & & & $0,60(0,45-0,81)$ & & \\
\hline \multicolumn{7}{|l|}{ Plano de saúde } \\
\hline Não & 1,00 & \#\# & & 1,00 & \#\# & \\
\hline Sim & $1,09(1,04-1,14)$ & & & $0,43(0,29-0,63)$ & & \\
\hline \multicolumn{7}{|c|}{ Consulta médica (últimos 15 dias) } \\
\hline Não & 1,00 & 1,00 & 0,011 & 1,00 & 1,00 & 0,003 \\
\hline Sim & $1,05(1,02-1,07)$ & $1,03(1,01-1,05)$ & & $0,51(0,35-0,75)$ & $0,57(0,39-0,82)$ & \\
\hline \multicolumn{7}{|c|}{ Internação hospitalar (últimos 12 meses) } \\
\hline Não & 1,00 & 1,00 & 0,001 & 1,00 & $\# \#$ & \\
\hline Sim & $1,08(1,05-1,11)$ & $1,02(1,01-1,05)$ & & $0,67(0,30-1,52)$ & & \\
\hline
\end{tabular}

IMC: índice de massa corporal.

* Valor de $p$ do teste de Wald;

** Variáveis que permaneceram na análise ajustada para controle de possíveis fatores de confusão;

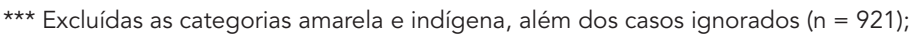

$\# \mathrm{n}=916$;

\#\# Excluído do modelo final de análise por apresentar $p>0,05$;

\#\#\# Excluída da análise multivariada por apresentar $p>0,20$ na análise não ajustada. 
o exame citopatológico; além disso, a realização dos exames pode ter sido superestimada por ser uma conduta adequada e esperada para as mulheres. O tempo transcorrido desde a realização do último exame também pode ter sido subestimado. Caplan et al. 13 evidenciaram que, embora as mulheres tendessem a subestimar o tempo desde a última realização do teste, o autorrelato é bastante preciso. Além disso, estudos nacionais e internacionais utilizam a informação autorreferida sobre a realização deste exame $7,10,11,14,15,16,17,18,19$.

Os resultados encontrados para a cobertura do exame de Papanicolaou na região urbana de Florianópolis corroboram dois estudos com mulheres de 25 a 59 anos de idade realizados nesta capital. O primeiro, realizado pelo Instituto $\mathrm{Na}$ cional de Câncer (INCA) em 2003, constatou a prevalência de $85,4 \%$ para a realização de pelo menos um exame nos três anos anteriores à pesquisa, estando associado positivamente à escolaridade 4 . A segunda pesquisa, realizada por meio de telefone, obteve $94,6 \%$ para a realização do exame em algum momento da vida, e $87,1 \%$ para a realização do exame nos últimos três anos 20 .

Estudos de base populacional conduzidos em diferentes momentos em uma cidade de médio porte do Rio Grande do Sul encontraram prevalências de exames de Papanicolaou em atraso, variando de $35 \%$ para mulheres com idade de 20 a 69 anos, em 1992 21, a 19\% para uma amostra de mulheres na faixa etária de 20 a 59 anos, em 2003 14. Também em 2003, em outra cidade deste estado, uma pesquisa realizada com uma amostra de mulheres sexualmente ativas, na faixa de 20 a 60 anos de idade, registrou 14,5\% de exames nunca realizados ou realizados há mais de três anos 15. Em São Paulo, estudo multicêntrico realizado entre 2001 e 2002 encontrou prevalência de $16,7 \%$ em mulheres com idade igual ou acima de 40 anos 16 . Porém, as pesquisas citadas examinaram mulheres em diferentes faixas de idade, dificultando a comparabilidade dos resultados.

Apesar de as prevalências de realização do Papanicolaou terem sido elevadas em Florianópolis, ainda existem desigualdades na distribuição desse exame na população feminina, com valores insatisfatórios para alguns estratos. Os principais gradientes encontrados, tanto para a realização do teste de Papanicolaou na vida, quanto para os exames em atraso, referem-se aos fatores demográficos, socioeconômicos e de uso dos serviços de saúde.

Em relação à idade, as mulheres mais jovens (20 a 29 anos) foram as que apresentaram a menor cobertura do Papanicolaou na vida e a maior proporção de exames em atraso, mantendo associação significativa somente para o primeiro desfecho. Esta situação pode ser explicada pela prioridade que o programa de prevenção do câncer de colo do útero tem dado às mulheres com idade entre 25 e 59 anos, não obstante as recomendações e garantia legal para sua realização por todas as mulheres que já iniciaram sua vida sexual 6,22 .

As mulheres que se declararam da cor preta apresentaram a menor cobertura na vida do teste de Papanicolaou e também a segunda maior prevalência de exames em atraso, no entanto a variável cor de pele não permaneceu associada aos respectivos desfechos após o ajuste pelas demais variáveis. Ainda assim, foi mantida nos respectivos modelos finais devido às evidencias na literatura de que a cor de pele está relacionada com desigualdades e iniquidades na utilização dos serviços de saúde e acesso a eles 23,24 . Esse achado é semelhante ao encontrado em estudos realizados nas regiões Sul e Nordeste do Brasil 15,25. Contrastando com esses resultados, em dois estudos nacionais, as mulheres da cor autorreferida como preta ou parda apresentaram maior prevalência de não realização do exame no intervalo de três anos, com significância estatística mesmo após o ajuste para escolaridade e/ ou idade 14,16.

Quanto ao estado civil, as mulheres que se declararam solteiras foram as que menos realizaram o exame na vida, com maior proporção de exames em atraso, evidenciando-se, mesmo após o ajuste pelas demais variáveis, como um fator relevante para os dois desfechos, principalmente em relação aos exames em atraso. Resultados semelhantes foram descritos por Martins et al. 11 em duas outras capitais brasileiras.

Ao se examinar a cobertura do Papanicolaou de acordo com a escolaridade, encontrou-se prevalência elevada para realização na vida inclusive entre as mulheres com menos anos de estudo. Contudo, estas apresentaram a maior proporção de exames em atraso, mesmo após o ajuste para as demais variáveis. Achados semelhantes foram descritos em estudos nacionais $11,14,16,17,25$ e internacionais 18,26 .

A literatura científica evidencia que menor renda familiar implica maiores taxas de não realização do exame de Papanicolaou 7,10,17. O presente estudo revelou maior cobertura na vida entre as mulheres com maior renda familiar per capita e maior prevalência de exames em atraso para aquelas com menor renda. O melhor nível socioeconômico e o conhecimento da periodicidade de realização do teste podem promover uma cobertura adequada e diminuir o risco de desenvolver o câncer cervical 19 .

Em relação ao estado de saúde e uso dos serviços de saúde, a presença de doenças crônicas 
e a consulta com médico nos 15 dias anteriores à entrevista favoreceram a regularidade do cuidado com realização de exames e a repetição destes com maior frequência e menor intervalo. Por outro lado, a internação hospitalar é um evento esporádico para a maioria das pessoas e favorece as investigações momentâneas, geralmente voltadas para a doença já instalada. Conforme encontrado em outras investigações 7,19, a probabilidade de receber cuidados preventivos em saúde é maior para as mulheres portadoras de doenças crônicas ou que receberam alguma intervenção, como consulta médica ou internação hospitalar, sugerindo que o rastreamento do câncer de colo do útero ocorre de forma oportunista. Neste tipo de rastreamento, a cobertura do Papanicolaou tem distribuição irregular, sendo realizado com uma frequência maior que a necessária e somente por algumas mulheres, conforme evidenciado por Quadros et al. 10 .

A disponibilidade de um plano privado de saúde favoreceu a melhor cobertura na vida do teste de Papanicolaou e o menor número de exames em atraso, porém este fator não permaneceu associado aos desfechos após o ajuste pelas demais variáveis. Destaca-se que o setor público foi responsável por menos da metade desse exame $(39,5 \%)$, todavia esses percentuais são cerca de cinco pontos superiores aos apontados em um inquérito nacional realizado pelo INCA 4 em 2004.

Outro aspecto que merece destaque é o fato de a visita do agente comunitário não influenciar na cobertura e manutenção da regularidade na coleta do teste de Papanicolaou. Situação semelhante também foi observada na Região Nordeste do país 25 , denunciando lacunas no cuidado integral da mulher na atenção básica. A Estratégia Saúde da Família tem como princípio a garantia de acesso à atenção básica, bem como o estabelecimento de vínculo e cuidado integral com a população adscrita. Neste aspecto, a atuação dos agentes de saúde é fundamental, contribuindo na identificação e captação das mulheres que deixam de realizar os exames preventivos do câncer de colo do útero 16 .

O presente estudo verificou que, no ano de 2009, a cobertura do exame de detecção precoce do câncer de colo de útero para as mulheres de 20 a 59 anos, residentes na área urbana de Florianópolis, foi dentro da meta preconizada pelo INCA. Apesar disso, evidenciou-se menor cobertura nos grupos sociais menos favorecidos, indicando a forte influência das disparidades socioeconômicas, demográficas e de uso dos serviços de saúde, assim como falta de continuidade no cuidado à saúde e nas ações de prevenção de câncer de colo uterino.

\section{Resumo}

A cobertura do teste de Papanicolaou e fatores associados foram investigados entre mulheres de 20 a 59 anos de idade, residentes na área urbana de Florianópolis, Santa Catarina, Brasil. Foi realizado estudo transversal de base populacional com amostra aleatória por conglomerados. Examinaram-se dois desfechos: realização do Papanicolaou em algum momento na vida e exames em atraso (período maior que três anos desde o último teste ou nunca realizado). Entre 952 mulheres entrevistadas, 93\% (IC95\%: 91,5-94,7) realizaram o teste pelo menos uma vez na vida e 14\% (IC95\%: 11,8-16,2) estavam com o procedimento em atraso. Por meio da regressão de Poisson, constatou-se que estado civil, escolaridade, doenças crônicas autorreferidas e consulta médica foram fatores significativamente associados com ambos os desfechos. Idade, renda e internação hospitalar no último ano estiveram associadas somente com a realização do Papanicolaou na vida. A cobertura do teste de Papanicolaou foi elevada, porém existem disparidades socioeconômicas e demográficas, além do predomínio do rastreamento oportunístico.

Esfregaço Vaginal; Neoplasias do Colo do Útero; Cobertura de Serviços de Saúde; Programas de Rastreamento 


\section{Colaboradores}

S. I. Gasperin participou da concepção do projeto, análise e interpretação dos dados e redação do artigo. A. F. Boing coordenou a pesquisa de campo e realizou a revisão crítica do conteúdo. E. Kupek orientou o projeto, a análise e a interpretação dos dados, realizou a revisão crítica do conteúdo e deu aprovação final para publicação.

\section{Agradecimentos}

Agradecemos à Dra. Nilza Nunes da Silva, Departamento de Epidemiologia da Faculdade de Saúde Pública da USP, pelo auxílio nos procedimentos da amostra. Aos técnicos do Instituto Brasileiro de Geografia e Estatística (IBGE) e da Secretaria Municipal de Saúde de Florianópolis, pelo auxílio na operacionalização deste estudo. Ao Conselho Nacional de Desenvolvimento Científico e tecnológico (CNPq; processo no ${ }^{\circ}$. 485327/2007-4).

\section{Referências}

1. Thun MJ, DeLancey JO, Center MM, Jemal A, Ward EM. The global burden of cancer: priorities for prevention. Carcinogenesis 2010; 31:100-10.

2. Boyle P, Levin B. World cancer report 2008. http:// www.iarc.fr/en/publications/pdfs-online/wcr/ 2008/wcr_2008.pdf/ (acessado em 03/Jun/2010).

3. Instituto Nacional de Câncer. Estimativas 2010: incidência de câncer no Brasil. http://www.inca.gov. br/estimativa/2010/ (acessado em 22/Mai/2010).

4. Instituto Nacional de Câncer. Inquérito domiciliar sobre comportamentos de risco e morbidade referida de doenças e agravos não transmissíveis: Brasil, 15 capitais e Distrito Federal, 2002-2003. Rio de Janeiro: Instituto Nacional de Câncer; 2004.

5. Boyle P. Current situation of screening for cancer. Ann Oncol 2002; 13 Suppl 4:189-98.

6. Ministério da Saúde. Controle dos cânceres do colo do útero e da mama. Brasília: Ministério da Saúde; 2006.

7. Dias-da-Costa JS, Olinto MTA, Gigante DP, Menezes AMB, Macedo S, Borba AT, et al. Cobertura do exame citopatológico na cidade de Pelotas, Rio Grande do Sul, Brasil. Cad Saúde Pública 2003; 19:191-7.

8. Vale DBAP, Morais SS, Pimenta AL, Zeferino LC. Avaliação do rastreamento do câncer do colo do útero na Estratégia Saúde da Família no Município de Amparo, São Paulo, Brasil. Cad Saúde Pública 2010; 26:383-90.
9. Martins LFL, Thuler LCS, Valente JG. Cobertura do exame de Papanicolaou no Brasil e seus fatores determinantes: uma revisão sistemática da literatura. Rev Bras Ginecol Obstet 2005; 27:485-92.

10. Quadros CAT, Victora CG, Dias-da-Costa JS. Coverage and focus of a cervical cancer prevention program in southern Brazil. Rev Panam Salud Pública 2004; 16:223-32.

11. Martins LFL, Valente JG, Thuler LCS. Factors related to inadequate cervical cancer screening in two Brazilian state capitals. Rev Saúde Pública 2009; 43:318-25.

12. Babor T, Higgins-Biddle J, Saunders JB, Monteiro MG. AUDIT, the Alcohol Use Disorders Identification Test: guidelines for use in primary care. Geneva: World Health Organization; 2001.

13. Caplan LS, McQueen DV, Qualters JR, Leff M, Garrett C, Calonge N. Validity of women's self-reports of cancer screening test utilization in a managed care population. Cancer Epidemiol Biomarkers Prev 2003; 12:1182-7.

14. Hackenhaar AA, Cesar JA, Domingues MR. Exame citopatológico de colo uterino em mulheres com idade entre 20 e 59 anos em Pelotas, RS: prevalência, foco e fatores associados à sua não realização. Rev Bras Epidemiol 2006; 9:103-11.

15. Muller DK, Dias-da-Costa JS, Luz AMH, Olinto MTA. Cobertura do exame citopatológico do colo do útero na cidade de São Leopoldo, Rio Grande do Sul, Brasil. Cad Saúde Pública 2008; 24:2511-20. 
16. Amorim VMSL, Barros MBA, César CLG, Carandina L, Goldbaum M. Fatores associados à não realização do exame de Papanicolaou: um estudo de base populacional no Município de Campinas, São Paulo, Brasil. Cad Saúde Pública 2006; 22:2329-38.

17. Cesar JA, Horta BL, Gomes G, Houlthausen RS, Willrich RM, Kaercher A, et al. Fatores associados à não realização de exame citopatológico de colo uterino no extremo Sul do Brasil. Cad Saúde Pública 2003 ; 19:1365-72.

18. Coughlin SS, King J, Richards TB, Ekwueme DU. Cervical cancer screening among women in metropolitan areas of the United States by individuallevel and area-based measures of socioeconomic status, 2000 to 2002. Cancer Epidemiol Biomarkers Prev 2006; 15:2154-9.

19. Pinho AA, França-Junior I, Schraiber LB, D'Oliveira AFPL. Cobertura e motivos para a realização ou não do teste de Papanicolaou no Município de São Paulo. Cad Saúde Pública 2003; 19 Suppl 2:S303-13.

20. Secretaria de Vigilância em Saúde, Ministério da Saúde. VIGITEL Brasil 2009. Vigilância de fatores de risco e proteção para doenças crônicas por inquérito telefônico. Brasília: Ministério da Saúde; 2010.

21. Dias-da-Costa JS, D'Elia PB, Manzolli P, Moreira MR. Cobertura do exame citopatológico na cidade de Pelotas, RS, Brasil. Rev Panam Salud Pública 1998; 3:308-13.
22. Brasil. Lei no. 11.664 de 29 de abril de 2008. Dispõe sobre a efetividade de ações de saúde que assegurem a prevenção, a detecção, o tratamento e o seguimento dos cânceres do colo uterino e de mama, no âmbito do Sistema Único de Saúde - SUS. http://www.planalto.gov.br/ccivil_03/_Ato20072010/2008/Lei/L11664.htm/ (acessado em 15/Jun/ 2009).

23. Fernandes LCL, Bertoldi AD, Barros AJD. Utilização dos serviços de saúde pela população coberta pela Estratégia de Saúde da Família. Rev Saúde Pública 2009; 43:595-603.

24. Lopes F. Para além da barreira dos números: desigualdades raciais e saúde. Cad Saúde Pública 2005; 21:1595-601.

25. Albuquerque KM, Frias PG, Andrade CLT, Aquino EML, Menezes G, Szwarcwald CL. Cobertura do teste de Papanicolaou e fatores associados à nãorealização: um olhar sobre o Programa de Prevenção do Câncer do Colo do Útero em Pernambuco, Brasil. Cad Saúde Pública 2009; 25 Suppl 2:S301-9.

26. Lockwood-Rayermann S. Characteristics of participation in cervical cancer screening. Cancer Nurs 2004; 27:353-63.

Recebido em 20/Dez/2010

Versão final reapresentada em 12/Abr/2011 Aprovado em 06/Mai/2011 